\title{
Papers
}

\section{Time trends in primary resistance to HIV drugs in the United Kingdom: multicentre observational study}

\author{
UK Group on Transmitted HIV Drug Resistance
}

\begin{abstract}
Objective To examine whether the level of primary resistance to HIV drugs is increasing in the United Kingdom.

Design Multicentre observational study.

Setting All virology laboratories in the United Kingdom carrying out tests for HIV resistance as part of routine clinical care.

Participants 2357 people infected with HIV who were tested for resistance before receiving antiretroviral therapy.

Main outcome measure Prevalence of drug resistance on basis of the Stanford genotypic interpretation system.

Results Over the study period (February 1996 to May 2003), 335 (14.2\%, 95\% confidence interval $12.8 \%$ to $15.7 \%)$ samples had mutations that conferred resistance to one or more antiretroviral drugs (9.3\% high level resistance, 5.9\% medium level resistance). The prevalence of primary resistance has increased markedly over time, although patterns are specific to drug class; the largest increase was for non-nucleoside reverse transcriptase inhibitors. In 2002-3, the prevalence of resistance to any antiretroviral drug to nucleoside or nucleotide reverse transcriptase inhibitors, to non-nucleoside reverse transcriptase inhibitors, or to protease inhibitors was $19.2 \%$ (15.7\% to $23.2 \%)$, $12.4 \%$ (9.5\% to $15.9 \%), 8.1 \%$ (5.8\% to $11.1 \%)$, and $6.6 \%$ (4.4\% to $9.3 \%)$, respectively. The risk of primary resistance was only weakly related to most demographic and clinical factors, including ethnicity and viral subtype.

Conclusions The United Kingdom has one of the highest reported rates of primary resistance to HIV drugs worldwide. Prevalence seems still to be increasing and is high in all demographic subgroups.
\end{abstract}

\section{Introduction}

Combination antiretroviral therapy has markedly improved the prognosis of patients infected with HIV. Concerns are mounting, however, about a secondary epidemic of drug resistant virus that would render treatment less effective. ${ }^{1-3}$ Previous studies on primary (transmitted) drug resistance in the United Kingdom either have been limited to a single centre or have focused on the relatively small number of patients identified shortly after becoming infected. ${ }^{45}$ We describe a national surveillance scheme for HIV drug resistance on the basis of routine clinical samples. Of about 13000 samples tested between 1996 and 2003, over 2300 were from patients who had never received antiretroviral therapy. We used these data to analyse the epidemiology of primary drug resistance in the United Kingdom, including temporal trends and associations with demographic and clinical factors.

\begin{abstract}
Methods
\section{Data on resistance}

The UK HIV drug resistance database was established in 2001 as a central repository of resistance tests carried out as part of routine clinical care in the United Kingdom. The tests in our analysis were based on DNA sequencing of the pol gene that were carried out in seven laboratories using a variety of in-house and commercial systems. All sequences encompassed at least codons 4-99 of the protease gene and 34-234 of the reverse transcriptase gene. We manually entered from the clinical resistance report all amino acid changes relative to a consensus or reference sequence. Other data items entered from the report included the clinical centre requesting the test, date of sample, date of test, and patient identifiers. Subtype was assigned using the STAR algorithm. ${ }^{6}$
\end{abstract}

\section{Clinical data}

We obtained demographic and clinical information, primarily to classify the patients' treatment status, from several sources. Electronic data were provided by the UK collaborative HIV cohort study (UK CHIC) ${ }^{7}$ and the UK register of HIV seroconverters. ${ }^{8}$ We sent a standard form to clinics that had carried out a minimum of 10 resistance tests, on which staff transcribed data from clinical notes. Information on exposure to antiretroviral therapy was sometimes given on request forms for resistance testing. Whenever possible we cross checked the information between these different sources. Finally, we defined a test as relating to recent infection if the patient was enrolled in the UK register of HIV seroconverters, which uses strict inclusion criteria, ${ }^{8}$ and the sample was taken within 18 months of a negative HIV antibody test result or other laboratory test result indicating acute infection.

To minimise bias from the misclassification of patients with experience of antiretroviral therapy as naïve to such therapy, we reviewed the resistance test request form or clinical notes held at the clinic to verify the information on antiretroviral therapy for a sample of patients with one or two major mutations ${ }^{9}$ and all patients with three or more major mutations. Information on antiretroviral therapy was incorrect in $26(18 \%)$ of the 142 cases checked; we excluded these patients from the analysis.

Tests included in analysis and definition of drug resistance The analysis includes all resistance tests in the database on patients aged over 16 years who were naïve to antiretroviral therapy. For patients tested more than once we included the first test only. We used the Stanford HIVdb algorithm (version

Members of the steering committees are on bmj.com 


\begin{tabular}{|c|c|c|c|c|c|}
\hline \multicolumn{2}{|c|}{$\begin{array}{l}\text { Nucleoside or nucleotide reverse transcriptase inhibitors } \\
(\mathrm{n}=233)\end{array}$} & \multicolumn{2}{|c|}{ Non-nucleoside reverse transcriptase inhibitors $(n=106)$} & \multicolumn{2}{|c|}{ Protease inhibitors $(\mathrm{n}=108)$} \\
\hline Mutation & No (\%) of samples* & Mutation & No $(\%)$ of samples* & Mutation & No $(\%)$ of samples* \\
\hline $41 \mathrm{~L}$ & $71(30)$ & $101 \mathrm{E} \dagger$ & $10(9)$ & $101 \dagger$ & $28(26)$ \\
\hline $44 \mathrm{D} \dagger$ & $10(4)$ & $103 \mathrm{~N}$ & $55(52)$ & $201 \dagger$ & $14(13)$ \\
\hline $67 \mathrm{~N}$ & $35(15)$ & $108 \mathrm{I \dagger}$ & $10(9)$ & $36 \mathrm{lt}$ & $50(46)$ \\
\hline 69D & $13(6)$ & $181 \mathrm{C}$ & $27(25)$ & 461 & $13(12)$ \\
\hline $69 \mathrm{N \dagger}$ & $25(11)$ & $190 \mathrm{~A}$ & $25(24)$ & $46 \mathrm{~L}$ & $13(12)$ \\
\hline $70 R$ & $29(12)$ & - & - & $54 \mathrm{~V}$ & $14(13)$ \\
\hline $118 \mathrm{It}$ & $29(12)$ & - & - & $63 \mathrm{Pt}$ & $54(50)$ \\
\hline $184 \mathrm{~V}$ & 39 (17) & - & - & $71 \mathrm{T \dagger}$ & 14 (13) \\
\hline $210 \mathrm{~W}$ & $24(10)$ & - & - & 71V† & $21(19)$ \\
\hline $215 \mathrm{C}$ & $18(8)$ & - & - & $77 \mathrm{It}$ & 36 (33) \\
\hline $215 \mathrm{D}$ & 33 (14) & - & - & $82 \mathrm{~A}$ & 16 (15) \\
\hline $215 \mathrm{~S}$ & $13(6)$ & - & - & $821 \dagger$ & $16(15)$ \\
\hline $215 Y$ & 28 (12) & - & - & $90 \mathrm{M}$ & $32(30)$ \\
\hline $219 \mathrm{E}$ & $11(5)$ & - & - & - & - \\
\hline $219 Q$ & $17(7)$ & - & - & - & - \\
\hline 333E† & $18(8)$ & - & - & - & - \\
\hline
\end{tabular}

Frequency of $333 \mathrm{E}$ may be underestimated as not all tests covered this codon.

*Percentage of total number of samples with resistance to that particular drug class.

†Mutations confer medium level resistance or high level resistance only in combination with other mutations.

2004.04) to assess the level of resistance to individual antiretroviral drugs. ${ }^{10}$ The algorithm is based on a matrix of scores for each drug-mutation combination; these are summed across all mutations in the test sample, and individual drug susceptibility is classified as "sensitive" (total score $<15$ ), "intermediate" (15-29), or "resistant" $(\geq 30)$. We refer to the last two categories as medium level resistance and high level resistance. The level of resistance to a specific drug class was defined as the highest level of resistance among all drugs within that class.

\section{Statistical methods}

We derived confidence intervals for proportions using the "exact" method, although these may underestimate the true uncertainty as sampling was not random. Logistic regression analysis was used to examine the association between demographic and clinical factors and the prevalence of resistance, adjusting for calendar year (fitted as a categorical variable) and centre; we combined centres with fewer than 10 patients. For CD4 count, we used the closest measurement within six months of the date of the resistance sample provided it preceded any use of antiretroviral therapy.

\section{Results}

A total of 2357 resistance tests on samples from antiretroviral therapy naive patients between February 1996 and May 2003 were available for analysis. One hundred and seventy two (7\%) samples were from patients known to have been recently infected at the time of testing. Overall, $116 \quad(4.9 \%, 95 \%$ confidence interval $4.1 \%$ to $5.9 \%$ ) samples showed medium level resistance and $219(9.3 \%, 8.1 \%$ to $10.5 \%)$ showed high level resistance to one or more drugs. When these categories were combined, reduced drug susceptibility was identified in 335 $(14.2 \%, 12.8 \%$ to $15.7 \%)$ samples. All further analyses are based on this inclusive definition of resistance unless stated otherwise.

\section{Patterns of resistance}

In total, $233(9.9 \%, 8.7 \%$ to $11.2 \%), 106(4.5 \%, 3.7 \%$ to $5.4 \%)$, and $108(4.6 \%, 3.8 \%$ to $5.5 \%)$ samples harboured mutations that cause resistance to nucleoside or nucleotide reverse transcriptase inhibitors, non-nucleoside reverse transcriptase inhibitors, and protease inhibitors, respectively. Most samples $(n=257 ; 10.9 \%$,
$9.7 \%$ to $12.2 \%$ ) were resistant to one drug class only (mostly nucleoside or nucleotide reverse transcriptase inhibitors); 44 $(1.9 \%, 1.4 \%$ to $2.5 \%)$ showed evidence of resistance to two classes and $34(1.4 \%, 1.0 \%$ to $2.0 \%)$ showed evidence of resistance to three classes.

Table 1 shows the most commonly observed mutations as highlighted by the Stanford HIVdb algorithm among the 335 resistant samples. Mutations at codon 215 in the reverse transcriptase gene including the reversion mutations T215C/D/ $\mathrm{E} / \mathrm{S}$, were most commonly observed; these changes were often associated with M41L. We did not observe K65R, I74V, or Y115F, associated with abacavir, didanosine, and tenofovir resistance. No mutations were detected associated with resistance to multinucleoside or nucleotide reverse transcriptase inhibitors, such as Q151M or insertions at codon 69. The most common non-nucleoside reverse transcriptase inhibitor mutation was K103N, which noticeably reduces the activity of all drugs within this class. Mutational patterns in protease were more complex, with a high frequency of several natural polymorphisms, which do not by themselves confer significant resistance.

Among individual nucleoside or nucleotide reverse transcriptase inhibitors drugs the prevalence of reduced drug susceptibility ranged widely from $2.5 \%$ (lamivudine) to $7.6 \%$ (zidovudine) (fig 1). The low level of resistance to lamivudine despite its widespread use provides indirect evidence that the lamivudine resistant virus is relatively "unfit." Due to strong cross resistance, we observed virtually identical figures for the non-nucleoside reverse transcriptase inhibitors delavirdine (4.1\%), efavirenz (4.2\%), and nevirapine (4.3\%). Resistance was generally least common among individual protease inhibitors, ranging from $2.1 \%$ (lopinavir) to $4.3 \%$ (nelfinavir).

Table 2 shows the most commonly prescribed first line antiretroviral therapy regimens in the six participating centres in the UK Collaborative HIV Cohort Study over the same period (February 1996 to May 2003) covered by our analysis. Overall, around $9 \%$ of first line regimens may have had reduced efficacy as a consequence of primary resistance $(6 \%$ under the stricter definition of high level resistance). Estimates depended on regimen and were comparatively high for the most popular regimens-zidovudine-lamivudine plus either efavirenz or nevirapine (11\% to $12 \%)$. 


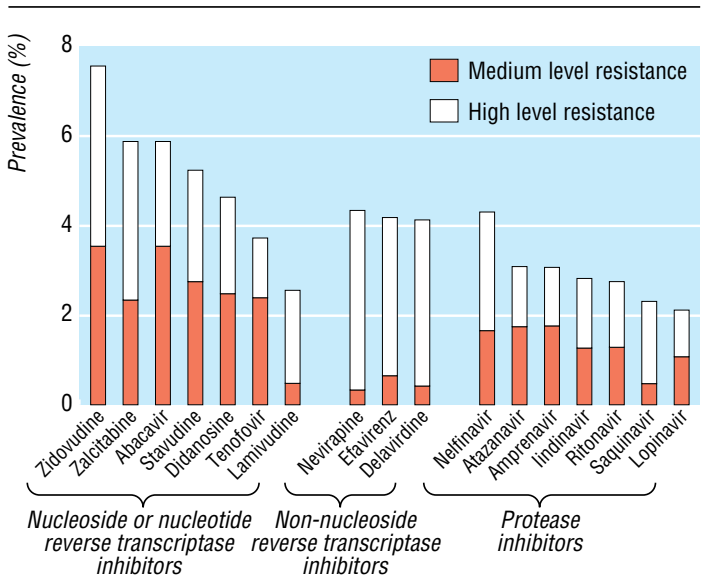

Fig 1 Prevalence of resistance to individual drugs

\section{Temporal trends}

The prevalence of primary drug resistance has increased noticeably, although patterns are specific to drug class and partly depend on whether medium level resistance is included (fig 2). The proportionate increase in resistance to nucleoside or nucleotide reverse transcriptase inhibitors was much smaller than for the non-nucleoside reverse transcriptase inhibitors or protease inhibitors, with some evidence of a levelling off in prevalence. In contrast, the rate of increase of resistance to non-nucleoside reverse transcriptase inhibitors has been approximately linear and has become as common as resistance to nucleoside or nucleoside reverse transcriptase inhibitors. The pattern for resistance to protease inhibitors was less clear: if medium level resistance is included, trends paralleled those of resistance to non-nucleoside reverse transcriptase inhibitors, whereas there was no indication of a recent increase if analysis was for high level resistance. In the most recent period for which data are available (2002-3), the prevalence of resistance to any antiretroviral drug, nucleoside or nucleotide reverse transcriptase inhibitors, non-nucleoside reverse transcriptase inhibitors, and protease inhibitors was 19.2\% (95\% confidence interval

Table 2 Prevalence of primary resistance to drugs for HIV comprising most common first line regimens used in UK collaborative HIV cohort study

\begin{tabular}{lccc} 
& $\begin{array}{c}\text { Percentage of } \\
\text { all first line } \\
\text { regimens } \\
\text { Regimen }\end{array}$ & $\begin{array}{c}\text { Prevalence (\%) of resistance to } \\
\text { one or more drugs in regiment }\end{array}$ \\
\cline { 3 - 5 } & $\begin{array}{c}\text { Medium level or } \\
\text { high level }\end{array}$ & $\begin{array}{c}\text { High level } \\
\text { resistance }\end{array}$ & resistance \\
\hline Zidovudine+lamivudine+efavirenz & 16.7 & 11.8 & 8.2 \\
\hline Zidovudine+lamivudine+nevirapine & 14.0 & 10.7 & 7.2 \\
\hline Didanosine+stavudine+nevirapine & 5.6 & 5.9 & 3.4 \\
\hline Stavudine+lamivudine+nevirapine & 5.3 & 6.4 & 3.4 \\
\hline Zidovudine+lamivudine+indinavir & 4.8 & 7.9 & 5.2 \\
\hline Zidovudine+lamivudine+abacavir & 3.8 & 9.2 & 5.7 \\
\hline Stavudine+lamivudine+efavirenz & 3.6 & 8.1 & 5.7 \\
\hline Zidovudine+lamivudine+nevirapine & 3.6 & 9.2 & 5.6 \\
\hline Stavudine+lamivudine+nevirapine & 3.4 & 7.5 & 5.1 \\
\hline Stavudine+lamivudine+indinavir & 3.4 & 6.2 & 2.3 \\
\hline Didanosine+stavudine+efavirenz & 2.3 & 7.1 & 4.5 \\
\hline Zidovudine+didanosine+nevirapine & 2.2 & 8.8 & 5.7 \\
\hline Zidovudine+lamivudine+abacavir+efavirenz & 2.1 & 12.5 & 9.1 \\
\hline Zidovudine+lamivudine+lopinavir & 2.0 & 10.3 & 6.3 \\
\hline
\end{tabular}

*Regimens with frequency of at least $2 \%$.

tBased on patterns observed in resistance database, assuming these were representative of all patients in study. Estimates are adjusted for variation in prevalence of resistance and use of different regimens (within annual time periods).
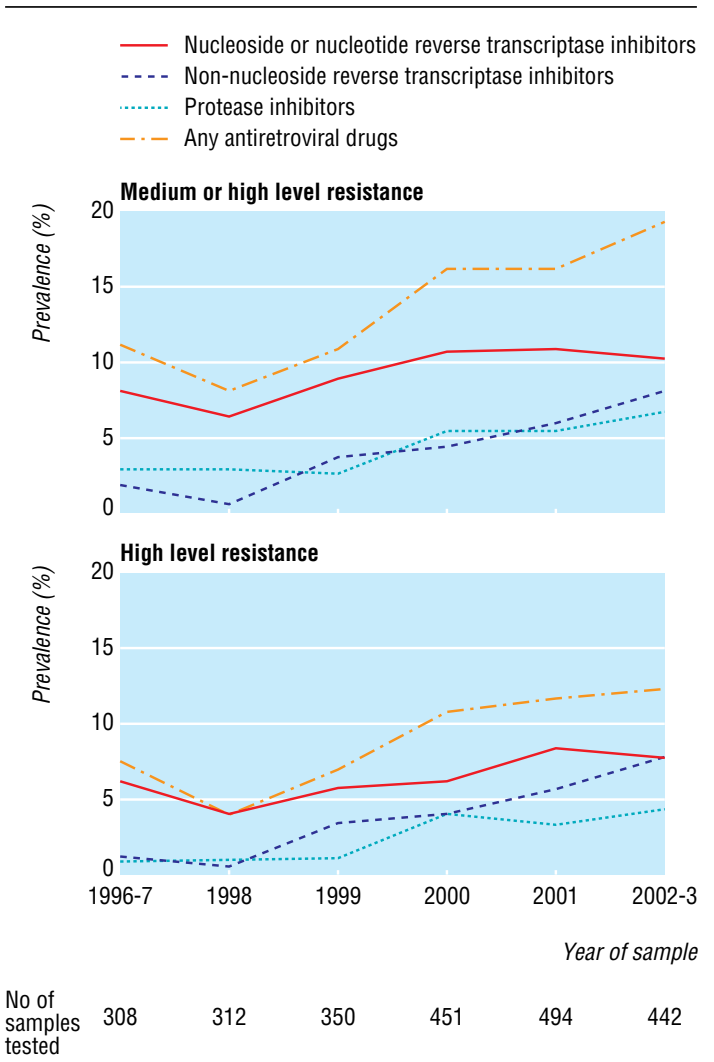

Fig 2 Prevalence of medium or high level drug resistance and high level drug resistance by calendar time. Values are number of samples tested at each time point

$15.7 \%$ to $23.2 \%), 12.4 \%$ (9.5\% to $15.9 \%$ ), $8.1 \%$ (5.8\% to $11.1 \%$ ), and $6.6 \%$ (4.4\% to $9.3 \%)$, respectively. Finally, we found primary resistance to at least one drug among an estimated 13\% of first line regimens started in 2002-3 in the UK collaborative HIV cohort study ( $9 \%$ for high level resistance only).

\section{Demographic and clinical factors}

Table 3 shows the prevalence of drug resistance according to selected demographic factors. Some of the crude comparisons are misleading because of confounding by calendar year and centre, and more reliable inference is through the odds ratios adjusted for these two factors. Mode of infection was not a strong risk factor apart from an apparently lower risk among injecting drug users. Associations with ethnicity and subtype of HIV, which are closely inter-related, were marginally significant. The rate of resistance was higher, although not substantially, in white patients and in those infected with subtype B virus. Patients younger than 30 experienced the highest level of resistance (odds ratio 1.32, 95\% confidence interval 1.02 to 1.72), although there was no clear trend across older age groups. The strongest determinant of primary resistance was acute infection: $22 \%$ of patients in this category had mutations conferring resistance compared with $14 \%$ of patients with an unknown duration of infection. In contrast, we found no apparent effect of CD4 count, a marker of the duration of infection, both overall and in a subanalysis excluding recently infected patients (data not shown).

\section{Discussion}

The prevalence of primary resistance to antiretroviral therapy in people infected with HIV in the United Kingdom is high in all 
Table 3 Effect of demographic and clinical factors on prevalence of primary resistance to HIV drugs in United Kingdom

\begin{tabular}{|c|c|c|c|c|c|}
\hline Factor & No of tests & $\begin{array}{l}\text { Prevalence of primary } \\
\text { resistance }(\%)^{*}\end{array}$ & Unadjusted odds ratio & Adjusted odds ratio $(95 \% \mathrm{Cl}) \dagger$ & $P$ value \\
\hline \multicolumn{6}{|l|}{ Year of sample: } \\
\hline $1996-7$ & 308 & 11.0 & 1.00 & 1.00 & \multirow[t]{6}{*}{$<0.001$} \\
\hline 1998 & 312 & 8.0 & 0.70 & 0.72 & \\
\hline 1999 & 350 & 10.9 & 0.98 & 0.93 & \\
\hline 2000 & 451 & 16.2 & 1.56 & 1.32 & \\
\hline 2001 & 494 & 16.2 & 1.56 & 1.36 & \\
\hline $2002-3$ & 442 & 19.2 & 1.92 & 1.76 & \\
\hline \multicolumn{6}{|l|}{ Location of clinic: } \\
\hline South east England & 1999 & 13.8 & 1.00 & 1.00 & \multirow[t]{2}{*}{0.82} \\
\hline Elsewhere in United Kingdom & 192 & 18.2 & 1.40 & 1.05 (0.70 to 1.58$)$ & \\
\hline \multicolumn{6}{|l|}{ Age (years): } \\
\hline $16-29$ & 583 & 17.0 & 1.00 & 1.00 & \multirow[t]{4}{*}{0.19} \\
\hline 30-34 & 661 & 13.5 & 0.76 & 0.77 (0.56 to 1.06$)$ & \\
\hline $35-39$ & 516 & 12.8 & 0.72 & 0.70 (0.49 to 0.99$)$ & \\
\hline$\geq 40$ & 592 & 13.7 & 0.77 & 0.80 (0.57 to 1.11$)$ & \\
\hline \multicolumn{6}{|l|}{ Probable mode of infection: } \\
\hline Male infected homosexually & 1487 & 13.5 & 1.00 & 1.00 & \multirow[t]{4}{*}{0.87} \\
\hline Male infected heterosexually & 161 & 14.9 & 1.12 & 0.87 (0.54 to 1.42$)$ & \\
\hline Female infected heterosexually & 248 & 16.5 & 1.27 & 0.92 (0.62 to 1.37$)$ & \\
\hline Injecting drug use & 51 & 8.8 & 0.70 & 0.76 (0.29 to 1.97$)$ & \\
\hline \multicolumn{6}{|l|}{ Ethnic group: } \\
\hline White & 1313 & 14.5 & 1.00 & 1.00 & \multirow[t]{5}{*}{0.03} \\
\hline Black African & 248 & 16.9 & 1.21 & 0.89 (0.60 to 1.32$)$ & \\
\hline Black Caribbean & 61 & 9.8 & 0.64 & 0.38 (0.15 to 0.96$)$ & \\
\hline Asian & 29 & 3.4 & 0.21 & 0.17 (0.02 to 1.34$)$ & \\
\hline Other or mixed & 104 & 12.5 & 0.84 & $0.70(0.37$ to 1.27$)$ & \\
\hline \multicolumn{6}{|l|}{ Subtype: } \\
\hline B & 1714 & 13.6 & 1.00 & 1.00 & \multirow[t]{6}{*}{0.05} \\
\hline C & 217 & 10.1 & 0.72 & 0.56 (0.34 to 0.91$)$ & \\
\hline$A$ & 102 & 13.7 & 1.01 & 0.80 (0.43 to 1.47$)$ & \\
\hline$A G$ & 50 & 22.0 & 1.79 & 1.08 (0.51 to 2.30$)$ & \\
\hline $\mathrm{D}$ & 39 & 10.3 & 0.73 & $0.63(0.22$ to 1.83$)$ & \\
\hline Other $(F, K, G, H, J)$ & 16 & 37.5 & 3.81 & 3.04 (1.02 to 9.02$)$ & \\
\hline \multicolumn{6}{|l|}{ Duration of HIV infection§: } \\
\hline Recent & 172 & 22.1 & 1.00 & 1.00 & \multirow[t]{2}{*}{0.006} \\
\hline Unknown & 2185 & 13.6 & 0.55 & 0.54 (0.35 to 1.27$)$ & \\
\hline \multicolumn{6}{|l|}{ CD4 count (cells/mm³): } \\
\hline $0-99$ & 271 & 11.4 & 1.00 & 1.00 & \multirow[t]{6}{*}{0.07} \\
\hline 100-199 & 260 & 17.3 & 1. 62 & 1.56 (0.94 to 2.59 ) & \\
\hline $200-299$ & 313 & 9.9 & 0.85 & 0.86 (0.50 to 1.48$)$ & \\
\hline 300-399 & 333 & 13.5 & 1.21 & 1.18 (0.71 to 1.97$)$ & \\
\hline $400-499$ & 272 & 17.3 & 1.62 & 1.69 (1.01 to 2.83 ) & \\
\hline$\geq 500$ & 481 & 13.1 & 1.17 & 1.26 (0.78 to 2.04$)$ & \\
\hline
\end{tabular}

Numbers do not always sum to total due to exclusion of small categories and missing data.

${ }^{*}$ Medium level and high level resistance combined.

†Adjusted for calendar year and centre, except location of clinic (adjusted for calendar year only) and year of sample (adjusted for centre only).

§See methods for definitions.

łLikelihood ratio test.

demographic subgroups and seems still to be increasing. As current potent regimens completely suppress viraemia in most patients, prevalence might have been expected to decrease. The fact that the opposite trend was observed implies there are important levels of transmission from patients who know that they are infected, pointing to the need for educational messages aimed at this group.

The definition of transmitted HIV drug resistance is highly problematic and the topic of ongoing debate. ${ }^{3}$ A central issue is how to distinguish natural polymorphisms from mutations that have arisen as a consequence of selective drug pressure in the individual who infected the index patient. Although this distinction is important from a public health perspective, it is less relevant for a prescribing clinician. We therefore used a genotypic interpretation system-the Stanford HIVdb algorithm-rather than the conventional definition of primary resistance, namely one or more drug associated mutations as defined by the International AIDS Society. ${ }^{9}$ A different interpretation system would have yielded different results. ${ }^{11}$ Further, the clinical significance of medium level resistance, which we included in our definition, is debatable.

\section{Temporal trends}

The overall prevalence of primary drug resistance in our study was $14 \%$, but this masks a strong underlying time trend with an estimated rate of $19 \%$ for the most recent time period (2002-3). Resistance to non-nucleoside reverse transcriptase inhibitors showed the sharpest increase, consistent with high levels of prescribing of this drug class in the United Kingdom, ${ }^{12}$ a high likelihood of developing resistance to this class, ${ }^{13}$ and trends in the 
prevalence of resistance observed in treated patients. ${ }^{14}$ Diverse and fluctuating temporal patterns in the rate of primary resistance to HIV have been reported in other studies, ${ }^{12}{ }^{15-17}$ although statistical variation is an important factor and mathematical models may give clearer insights into underlying trends. The cost effectiveness of testing for drug resistance before therapy is highly sensitive to the prevalence of primary resistance. A panel of European experts recommended testing the earliest available blood sample if suspicion of resistance is high for that individual or if the prevalence in that population exceeds $10 \% \cdot{ }^{18}$ Our estimate of the overall prevalence in 2002-3 was almost double this figure. However, this signifies resistance to any one of 17 antiretroviral drugs, whereas only a limited number of drugs tend to be used in first line regimens. Using data from the UK collaborative HIV cohort study, we estimated that $13 \%$ of first line regimens started in 2002-3 may have been suboptimal due to resistance to one or more drugs in the regimen.

\section{Demographic factors}

The higher level of primary resistance in younger patients warrants further investigation, although another study found that age was only weakly related to HIV incidence among homosexual men attending clinics for sexually transmitted infections. ${ }^{19}$ Lower prevalence rates were observed in Asian and Caribbean patients and those infected through injecting drug use, although this finding should be interpreted cautiously because of the small number of samples tested in these groups. African patients, who comprise around $45 \%$ of new diagnoses of HIV-1 infection in the United Kingdom, ${ }^{20}$ had a similar prevalence to white patients. This finding was unexpected as most of the infections are likely to have been acquired in Africa, where access to antiretroviral therapy has been limited.

As expected, primary drug resistance was significantly more common in patients with recent HIV infection (22\%) than in those with presumed chronic infection (14\%). This is explained by the fact that those with presumed chronic infection will have been infected on average several years earlier, when the transmission of resistant viral strains was less common, and the longer opportunity for reversion of resistant viral mutations. ${ }^{3}$ The validity of using chronically infected patients to measure transmitted drug resistance has been widely discussed. ${ }^{3}$ The clear and biologically plausible time trends we observed among this group suggests that they do provide useful insights into the epidemiology of primary drug resistance.

\section{Methodological considerations}

Comparisons with other studies are complicated by the use of different definitions of HIV resistance. With this caveat, our rate of primary resistance (14\%) is considerably higher than estimates in chronically infected patients reported from the United States $(7 \%),{ }^{21}$ France $(6 \%),{ }^{22}$ and elsewhere in Europe $(10 \%) .{ }^{17}$ We carefully considered the possibility of bias in our estimates. Resistance testing was ad hoc over the period of the study and patients may have been selectively tested if there was a suspicion they had been infected by someone who had received antiretroviral therapy. The potential for such a bias is strong: the ratio of the number of resistance tests in patients naive to antiretroviral therapy (2357) to the number of new diagnoses in the United Kingdom over the same period (about 29000 ) was only 1:12. Furthermore, an estimated one third of HIV infections are thought to be undiagnosed. One of the centres (Chelsea and Westminster) routinely tests samples before therapy; here the prevalence of resistance $(11.2 \%)$ was significantly lower than in other centres (odds ratio adjusted for calendar year $=0.61$,
What is already known on this topic

Primary HIV drug resistance limits therapeutic options

Its spread could negate the large reductions in morbidity and mortality since combination antiretroviral therapy was introduced

Knowledge on the level and patterns of primary drug resistance in the United Kingdom is limited

\section{What this study adds}

The United Kingdom has one of the highest reported rates of primary resistance to HIV drugs

Prevalence seems to be increasing and is high in all demographic subgroups

$\mathrm{P}<0.001)$. Although seeming to show the presence of selection bias, this also confirms a high rate of resistance among unselected patients. Finally, estimates of the level of primary resistance should become increasingly accurate if, as is now recommended, resistance testing before therapy becomes a standard clinical investigation.

Another potential bias arises from the misclassification of treatment experienced patients as treatment naive. Firstly, treatment status was largely inferred from information from local electronic clinical databases, which are prone to inaccuracy. Secondly, many patients received care at two or more sites, and although we attempted to link their records we may not always have been successful. Thirdly, some treatment experienced patients may have denied having received antiretroviral therapy. ${ }^{23}$ We carried out various checks to eliminate these sources of error as far as possible. Also, treatment misclassification bias is unlikely in acutely infected patients, yet the prevalence of primary resistance in this group was consistent with the level in chronically infected patients, allowing for a general increase over calendar time.

\section{Conclusion}

Collating the results of routine HIV drug resistance tests can provide valuable insights into the population spread of drug resistant HIV at small additional cost. The prevalence of primary resistance in the United Kingdom is high in all demographic subgroups and seems still to be increasing. By limiting the therapeutic options for a significant number of patients, the secondary epidemic of drug resistant HIV represents a major clinical and public health problem.

The UK Group on Transmitted HIV Drug Resistance is a collaboration between the UK Collaborative Group on HIV Drug Resistance, the UK Collaborative HIV Cohort Study, and the UK Register of HIV Seroconverters. Members of the writing group are Patricia Cane, Ian Chrystie, David Dunn, Barry Evans, Anna Maria Geretti, Hannah Green, Andrew Phillips, Deenan Pillay, Kholoud Porter, Anton Pozniak, Caroline Sabin, Erasmus Smit, Jonathan Weber, and Mark Zuckerman; affiliations are on bmj.com. We thank Tommy Lui (Stanford) and Patrick Woodburn for help with various aspects of the analysis, and the clinicians, virologists, data managers, and research nurses in participating centres who assisted with the provision of data.

Contributors: All authors were involved in setting up the UK HIV drug resistance database, one of the principal aims of which was to monitor transmitted drug resistance. CS coordinates the UK collaborative HIV cohort study and KP coordinates the UK register of HIV seroconverters. DP, AP, PC, RS, MZ, JW, AMG, and IC facilitated the provision of genotypic resistance data. DD and HG carried out the statistical analysis. DD wrote 
and edited drafts of the paper. All other authors made substantive comments on drafts of the paper. DD is guarantor.

Funding: The UK HIV drug resistance database is funded by the Department of Health; the views expressed in the publication are those of the authors and not necessarily those of the Department of Health. UK CHIC (grant G0000199) and the UK register of HIV seroconverters (grant G9324150) are funded by the Medical Research Council, United Kingdom. Competing interests: None declared.

Ethical approval: This study was approved by the UK multicentre research ethics committee and relevant local research ethic committees.

1 Little SJ, Holte S, Routy JP, Daar ES, Markowitz M, Collier AC, et al. Antiretroviral-drug resistance among patients recently infected with HIV. N Engl J Med 2002;347:385-94.

2 Grant RM, Hecht FM, Warmerdam M, Liu L, Liegler T, Petropoulos CJ, et al. Time trends in primary HIV-1 drug resistance among recently infected persons. JAMA 2002;288:181-8.

3 Eron JJ. The role of resistance testing in treatment-naive HIV-infected individuals. http://www.clinicaloptions.com/hiv/treatment/testing/\#eron (accessed 1 May 2005).

4 UK Collaborative Group on Monitoring the Transmission of HIV Drug Resistance Analysis of prevalence of HIV-1 drug resistance in primary infections in the United Kingdom. BMJ 2001;322:1087-8.

5 Geretti AM, Smith M, Osner N, O'Shea S, Chrystie I, Easterbrook P, et al. Prevalence of antiretroviral resistance in a South London cohort of treatment-naive HIV-1-infected patients. AIDS 2001:15:1082-4.

6 Gale CV, Myers R, Tedder RS, Williams IG, Kellam P. Development of a novel human immunodeficiency virus type 1 subtyping tool, subtype analyzer (STAR): analysis of subtype distribution in London. AIDS Res Hum Retroviruses 2004;20:457-64.

7 The UK Collaborative HIV Cohort Steering Committee. The creation of a large UK-based multicentre cohort of HIV-infected individuals: the UK collaborative HIV cohort (UK CHIC) study. HIV Med 2004:5:115-24.

8 UK Register of HIV Seroconverters Steering Committee. The AIDS incubation period in the UK estimated from a national register of HIV seroconverters. AIDS in the UK est

9 Johnson VA, Brun-Vezinet F, Clotet B, Conway B, D'Aquila RT, Demeter LM, et al. Update of the drug resistance mutations in HIV-1: 2004. Top HIV Med 2004;12:119-24 10 Release notes for HIVseq, HIVdb, HIValg. http://hivdb.stanford.edu/pages/asi/ releaseNotes/ (accessed 1 May 2005).

11 De Luca A, Cozzi-Lepri A, Perno CF, Balotta C, Di Giambenedetto S, Poggio A, et al Variability in the interpretation of transmitted genotypic HIV-1 drug resistance and prediction of virological outcomes of the initial HAART by distinct systems. Antivi Ther 2004;9:743-52.
12 Sabin CA, Hill T, Lampe F, Matthias R, Bhagani S, Gilson R, et al. Treatment exhaustion of highly active antiretroviral therapy (HAART) among individuals infected with HIV in the United Kingdom: multicentre cohort study. BMJ 2005;330:695-8.

13 UK Collaborative Group on HIV Drug Resistance and UK CHIC Study Group. Long term probability of detection of HIV-1 drug resistance after starting antiretroviral therapy in routine clinical practice. AIDS 2005;19:487-94.

14 UK Collaborative Group on HIV Drug Resistance. Estimating HIV-1 drug resistance in antiretroviral treated individuals within the UK. J Infect Dis 2005;192:967-73.

15 Yerly S, Vora S, Rizzardi P, Chave JP, Vernazza PL, Flepp M, et al. Acute HIV infection: impact on the spread of HIV and transmission of drug resistance. AIDS 2001:15:228792.

16 Ammaranond P, Cunningham P, Oelrichs R, Suzuki K, Harris C, Leas L, et al. No increase in protease resistance and a decrease in reverse transcriptase resistance mutations in primary HIV-1 infection: 1992-2001. AIDS 2003;17:264-7.

17 Wensing AM, van de Vijver DA, Angarano G, Asjo B, Balotta C, Boeri E, et al. Prevalence of drug-resistant HIV-1 variants in untreated individuals in Europe: implications for clinical management. J Infect Dis 2005;192:958-66.

18 Vandamme AM, Sonnerborg A, Ait-Khaled M, Albert J, Asjo B, Bacheler L, et al. Updated European recommendations for the clinical use of HIV drug resistance testing. Antivir Ther 2004;9:829-48.

19 Murphy G, Charlett A, Jordan LF, Osner N, Gill ON, Parry JV. HIV incidence appears constant in men who have sex with men despite widespread use of effective antiretroviral therapy. AIDS 2004;18:265-72.

20 HIV and AIDS in the United Kingdom quarterly update: data to the end of December 2004. Commun Dis Rep CDR Wkly 2005;15(8).

21 Bennett DE, Zaidi IF, Heneine W, Woods T, Garcia-Lerma JG, Smith AJ, et al Prevalence of mutations associated with antiretroviral drug resistance among men and women newly diagnosed with HIV in 10 US cities, 1997-2001. Antivir The 2003;8:S133

22 Chaix ML, Descamps D, Mouajjah S, Deveau C, Andre P, Cottalorda J, et al. French national sentinel survey of antiretroviral resistance in patients with HIV-1 primary infection and in antiretroviral-naive chronically infected patients in 2001-2002. Antivir Ther 2003;8:S137.

23 Foley E, Watson-Jones D, Loveday C. Extensive antiretroviral therapy resistance in an HIV-infected Zimbabwean patient in the UK. AIDS 2003;17:2404-5.

(Accepted 27 September 2005)

doi 10.1136/bmj.38665.534595.55

Medical Research Centre Clinical Trials Unit, London NW1 2DA David Dunn senior scientist

Correspondence to: D Dunn d.dunn@ctu.mrc.ac.uk 